\title{
Numerical simulation of wellbore and formation temperature fields in carbonate formations during drilling and shut-in in the presence of lost circulation
}

\author{
Pan Baozhi'*, Li Ding ${ }^{1}$, Chen Gang ${ }^{1}$, Wang Qincong ${ }^{2}$, Ma Lixin ${ }^{2}$ and Liu \\ Sihui ${ }^{1}$ \\ ${ }^{1}$ College of Geo-Exploration Science and Technology, Jilin University, Changchun, Jilin 130026, China \\ ${ }^{2}$ Xi'an Research Institute of China Coal Technology \& Engineering Group Corp, Xi'an, Shaanxi 710077, China \\ ${ }^{3}$ Northwest Oil and Gas Branch Sinopec, Urumqi, Xinjiang 830011, China \\ (C) China University of Petroleum (Beijing) and Springer-Verlag Berlin Heidelberg 2014
}

\begin{abstract}
Temperature curves reflect geothermal gradients and local temperature anomalies, thus providing a new understanding of the underground reservoir conditions. When encountering caverns or fractures and fissures during drilling, lost circulation may occur and result in a change to the original formation temperature field, and in severe cases, even the conventional open hole well logging data cannot be obtained. This paper uses finite element analysis software COMSOL to establish a heat transfer model for the wellbore/reservoir formation system during drilling and shut-in in the presence of lost circulation, and a case study is made in a carbonate reservoir in the Tahe oilfield. On the basis of the above, we analyze the temperature distribution in the leakage zone, and the studies have shown that the leakage and petrophysical properties have an impact on the temperature of the wellbore and formation, hence we can estimate the reservoir permeability using the temperature data. In addition, the determination of the temperature recovery time after some drilling fluids have leaked into the formation will help in recognizing the subsurface temperature field of the carbonate formation correctly, thus enhancing production logging interpretation accuracy and improving the understanding of later measurements.
\end{abstract}

Key words: Temperature field, numerical simulation, finite element, drilling fluid invasion, leakage

\section{Introduction}

$70 \%$ of the world's hydrocarbon resources are found in carbonate formations which are often deeply buried, strongly heterogeneous, and have high-salinity formation water (Dou et al, 2011; Erik et al, 2011). Fractured-cavernous reservoirs in the Tahe oilfield are characterized by strong heterogeneity, hence lost circulation often occurs when the drill bit encounters natural fissures, fractures, or caverns, and the drilling fluid flows into the newly available space, thus the well must be rapidly completed and put into production. The drilling fluid loss may affect log measurements and even the safety of drilling operation in severe leakage zones in the reservoir, resulting in the lack of open hole well logging data (Chin, 2002). Therefore, it is important to identify leakage zones and to simulate the variation of the formation temperature, thus providing evidence for the original formation temperature and temperature logging results, which are affected by drilling fluid invasion. The understanding of temperature logging data will be a profitable supplement

*Corresponding author. email: panbaozhi@jlu.edu.cn

Received May 16, 2013 when the other logging information is unavailable, especially in the wells which must be rapidly completed and put into production during lost circulation.

In 1962, Ramey established a function of wellbore temperature, depth and production time, which is known as Ramey's equation. This equation assumed that the fluid flows only in the tubing and casing, and does not flow into the formation (Ramey, 1962; Atkinson et al, 1978). In 1991, Beirute simulated temperature profiles in wells during fluid circulation, cementation and shut-in, respectively (Beirute, 1991). In 1996, Feng et al established the temperature fields of wells and formations, but it was not a real multifield coupled model (Feng et al, 1996). Xu and Cui (1996) established a well temperature field model using twophase flow theory and heat transfer theory, and solved the temperature fields of wellbores and formations by coupling iteration, but this model did not consider convective heat transfer. Conventional simulations of temperature fields mostly use the finite difference method, and research into the finite element method of solving temperature fields has yet to be perfected. In this paper, we use the finite element software COMSOL to solve the problems of velocity field and temperature field, assuming that the fluid in the well is 
pipe flow, which can be described by the Navier-Stokers (NS) equation, and the fluid in the formation is laminar flow, which can be calculated by Darcy's equation. By applying this method, the velocity field is of more practical significance. In this paper we predict the drilling fluid temperature and discuss the effect of petrophysical properties of reservoirs on drilling fluid and formation temperatures. In addition, we also simulate the temperature fields of the leakage zones in the carbonate reservoir.

\section{Mathematical modeling}

In numerical simulation of the temperature field, in addition to the alteration of fluid flow caused by the formation temperature change, the heat transfer in porous media caused by fluid flow should also be taken into account. Meanwhile, the stress field affects the flow field due to the changes of reservoir permeability and porosity (Zeiser and Durst, 2005; Georgiadis et al, 1999; Gao et al, 2012). Therefore, the drilling fluid invasion and lost circulation is a multifield coupling problem of temperature, pressure and velocity fields in porous media (Sheng et al, 2009). For modeling, we assume that:

1) The formation is homogenous and isotropic.

2) The thermodynamic parameters of the medium (such as thermal conductivity, specific heat capacity, etc.) do not change with temperature.

\subsection{Temperature field modeling}

Taking the wellbore and the surrounding formation as the research object, the entire system follows the energy conservation equation in the process of drilling fluid invasion (Zhang et al, 2011), as shown in Eq. (1), where velocity $\mathbf{u}$ is calculated by the velocity field of the percolation model:

$$
\left\{\begin{array}{l}
(\rho C)_{\mathrm{eq}} \frac{\partial T}{\partial t}+\rho C \mathbf{u} \cdot \nabla T=\nabla \cdot\left(k_{\mathrm{eq}} \nabla T\right)+Q \\
k_{\mathrm{eq}}=(1-\varphi) k_{\mathrm{p}}+\varphi k \\
(\rho C)_{\mathrm{eq}}=(1-\varphi) \rho_{\mathrm{p}} C_{\mathrm{p}}+\varphi \rho C
\end{array}\right.
$$

where $k$ and $k_{\mathrm{p}}$ are the thermal conductivity coefficients of the fluid and the matrix, $\mathrm{W} \cdot \mathrm{m}^{-1} \cdot{ }^{\circ} \mathrm{C}^{-1} ; k_{\mathrm{eq}}$ is the equivalent thermal conductivity coefficient, $\mathrm{W} \cdot \mathrm{m}^{-1} \cdot{ }^{\circ} \mathrm{C}^{-1} ; C$ and $C_{\mathrm{p}}$ are the specific heat capacity of the fluid and the matrix, $\mathrm{kJ}^{\mathrm{J}} \cdot \mathrm{kg}^{-1} \cdot{ }^{\circ} \mathrm{C}^{-1} ; \rho$ and $\rho_{\mathrm{p}}$ are the density of the fluid and the matrix, $\mathrm{kg} \cdot \mathrm{m}^{-3} ; \varphi$ is the formation porosity; and $Q$ is the heat source, $\mathrm{W} \cdot \mathrm{m}^{-3}$. The initial and boundary conditions needed to solve Eq. (1) are as follows (Xu, 1994):

1) Initial condition:

The initial temperatures of the formation and the wellbore are the temperatures measured under static state conditions:

$$
\left.T\right|_{t=0}=T_{z=0}+G_{T} z
$$

2) Boundary condition:

The upper and bottom boundaries of the formation are adiabatic:

$$
\left.\lambda \frac{\partial T}{\partial Z}\right|_{z=z_{\max } ; r>r_{\mathrm{b}}}=\left.\lambda \frac{\partial T}{\partial Z}\right|_{z=0 ; r>r_{\mathrm{b}}}=0
$$

The heat is transferred by convection between the wellbore and the formation:

$$
-\left.k \frac{\partial T}{\partial n}\right|_{\Gamma}=\left.\alpha\left(T-T_{\mathrm{f}}\right)\right|_{\Gamma}
$$

where $G_{\mathrm{T}}$ is the geothermal gradient, ${ }^{\circ} \mathrm{C} / 100 \mathrm{~m}$; $\alpha$ is the convection heat transfer coefficient of the formation, $\mathrm{W} \cdot\left(\mathrm{m}^{2} \cdot{ }^{\circ} \mathrm{C}\right)^{-1}$; and $T_{\mathrm{f}}$ is the drilling fluid temperature, ${ }^{\circ} \mathrm{C}$.

\subsection{Fluid-solid coupling and seepage modeling}

The fluid flow in the wellbore is affected by stress, where we may describe this conduit flow by the Navier-Stokers (NS) equation (Liu and Liang, 2009; Liang, 2013; Fang and $\mathrm{Zi}, 2013$ ), and use Darcy's equation to describe the migration of fluid in the porous medium. In this paper, we combine Darcy's equation (Baber et al, 2012) and the NS equation to solve the coupling problem of the fluid flow in wellbore and porous media.

The NS equation describing fluid flow in the wellbore can be written as follows:

$$
\rho_{1} \frac{\mathrm{d} u}{\mathrm{~d} t}=\rho_{1} \mathbf{F}+\eta_{1} \nabla^{2} \mathbf{u}-\nabla P_{1}
$$

where $\mathbf{F}$ represents the external stress, $\mathrm{N} \cdot \mathrm{m}^{-3}$, which can be expressed as follows:

$$
\left(F_{x}, F_{y}, F_{z}\right)=\left(-\frac{\partial P_{1}}{\partial x}+\rho g_{x},-\frac{\partial P_{1}}{\partial y}+\rho g_{y},-\frac{\partial P_{1}}{\partial z}+\rho g_{z}\right)
$$

where $P_{1}$ is the drilling fluid pressure, $\mathrm{Pa}$; $\mathbf{u}$ is the velocity vector, $\mathrm{m} \cdot \mathrm{s}^{-1} ; \rho_{1}$ is the drilling fluid density, $\mathrm{kg} \cdot \mathrm{m}^{-3} ; \eta_{1}$ is the drilling fluid viscosity, $\mathrm{Pa} \cdot \mathrm{s} ; \mathrm{g}$ is gravity acceleration, $\mathrm{m} \cdot \mathrm{s}^{-2}$.

The fluid flow in the reservoir is described by Darcy's equation:

$$
\left\{\begin{array}{l}
\frac{\partial}{\partial t}\left(\rho_{2} \varphi\right)+\nabla \cdot\left(\rho_{2} \mathbf{u}\right)=Q_{\mathrm{m}} \\
\frac{\partial}{\partial t}\left(\rho_{2} \varphi\right)=\rho_{2}\left[\varphi \chi_{\mathrm{f}}+(1-\varphi) \chi_{\mathrm{p}}\right] \frac{\partial P_{2}}{\partial t} \\
\mathbf{u}=-\frac{K_{\mathrm{m}}}{\eta_{2}} \nabla P_{2}
\end{array}\right.
$$

where $P_{2}$ is the reservoir pressure, $\mathrm{Pa} ; \rho_{2}$ is the reservoir fluid density, $\mathrm{kg} \cdot \mathrm{m}^{-3} ; \chi_{\mathrm{f}}$ and $\chi_{\mathrm{p}}$ are the effective compression coefficient of the fluid and the matrix, $\mathrm{m} \cdot \mathrm{S}^{2} \cdot \mathrm{kg}^{-1} ; \eta_{2}$ is the formation fluid viscosity, $\mathrm{Pa} \cdot \mathrm{s} ; K_{\mathrm{m}}$ is the reservoir permeability, $10^{-3} \mu \mathrm{m}^{2} ; \varphi$ is the reservoir porosity.

The conditions for solving Eqs. (5) and (7) are as follows (Ji et al, 2011):

1) Initial condition:

The wellbore pressure is $P_{0}$ when $t=0$, i.e.

$$
\left.P(x, y, z, t)\right|_{t=0}=P_{0}
$$

2) Boundary condition:

The pressure applied on the wellbore wall is $P_{0}$; and the reservoir pressure in the infinite distance is $P_{1}$.

$$
\left\{\begin{array}{l}
\left.P(x, y, z, t)\right|_{l=0}=P_{1} \\
\left.P(x, y, z, t)\right|_{\infty}=P_{0}
\end{array}\right.
$$




\subsection{Model establishment and simulation}

We used COMSOL MULTIPHYSICS software to solve the heat transfer equation and flow equation in porous media using a thermal-fluid coupling method (Liu and Wang, 2008). First, we construct a geometrical model of the real formation. Then, we select a physical field equation which is needed in simulation. We set the initial formation temperature, initial circulation rate of the drilling fluid, as well as the physical properties of the formation such as porosity, permeability and heat transfer coefficient based on the log data and local temperature gradient (Saenger et al, 2011). Next we perform mesh generation for the geometrical model. Refined grids on the wellbore wall are needed in order to improve the calculation accuracy and efficiency. Finally, we run the simulation. The reservoir parameters and temperature are checked to determine whether the simulation has converged. If not, an additional iteration is conducted, in which the nonlinear coefficients are updated and the tolerances are tightened, until the specified target convergence condition is achieved (Lu and Wheeler, 2009; Lacroix et al, 2003; Sun et al, 2012).

\section{Results and discussion}

\subsection{Numerical simulation of the temperature field during drilling fluid invasion}

Some drilling fluid may invade the formation during drilling, which causes changes in the temperatures of the surrounding formation and the drilling fluid in the wellbore and reservoir (Ning et al, 2013; Poulsen et al, 2012). We establish a model with a wellbore radius of $0.2 \mathrm{~m}$, and the formation surrounding the wellbore is $4 \mathrm{~m}$ in the radial direction and $2 \mathrm{~m}$ in thickness. The wellbore is filled with the drilling fluid, and the formation is porous, with a porosity of 0.05 . Assuming that the wellbore fluid is characterized by free flow, which is described by Eq. (5), and the fluid flow in the reservoir is described by Darcy's equation, which is shown in Eq. (7), the temperature fields of the wellbore and formation follow energy conservation, shown in Eq. (1).

We take well $\mathrm{Y}$ in the Tahe oilfield as an example, in which the initial temperatures of the drilling fluid and the formation are $50{ }^{\circ} \mathrm{C}$ and $126.6{ }^{\circ} \mathrm{C}$ respectively. Fig. 1 shows the temperature profile changing with time. We simulate the temperature-time profiles of the drilling fluid and the formation with different porosities using this model, as shown in Fig. 2. During drilling, the drilling fluid invades the formation, then heat transfer occurs, and the temperature of the drilling fluid in the wellbore gradually rises after drilling fluid circulation is halted, while the formation temperature decreases rapidly to $116{ }^{\circ} \mathrm{C}$ and then increases to its initial value. Fig. 2(a) is the temperature field of the wellbore and its surrounding formation (the origin at the well center with coordinates of $(0,0)$ ). The drilling fluid temperature increases rapidly in the first two days, then reaches a constant when it is close to the formation temperature. The drilling fluid temperature increases quickly in low porosity reservoirs. The lower the porosity, the smaller the amount of drilling fluid which invades the formation, and the faster the heat transfers between the drilling fluid and the reservoir. Fig. 2(b) is the reservoir temperature at coordinates of $(0.3,0)$, which is $0.3 \mathrm{~m}$ away from the borehole axis. The heat is continuously transferred from the formation to the drilling fluid, so the formation temperature decreases quickly then recovers slowly to its initial value. At first five hours (see the small diagram in Fig. 2(b)), we can see that the high porosity reservoir temperature reduces rapidly, but the temperature recovery process is relatively slow, which is due to the fact that invasion is more likely to occur in high permeability formations.

We then simulate the change of temperature with depth in the formation after invasion, shown in Fig. 3. The formation temperature in Well Y (a depth of 5,680-5,700 m) was measured after the well was shut in for 30 days. It can be seen that the numerical results agree well with the measured values.

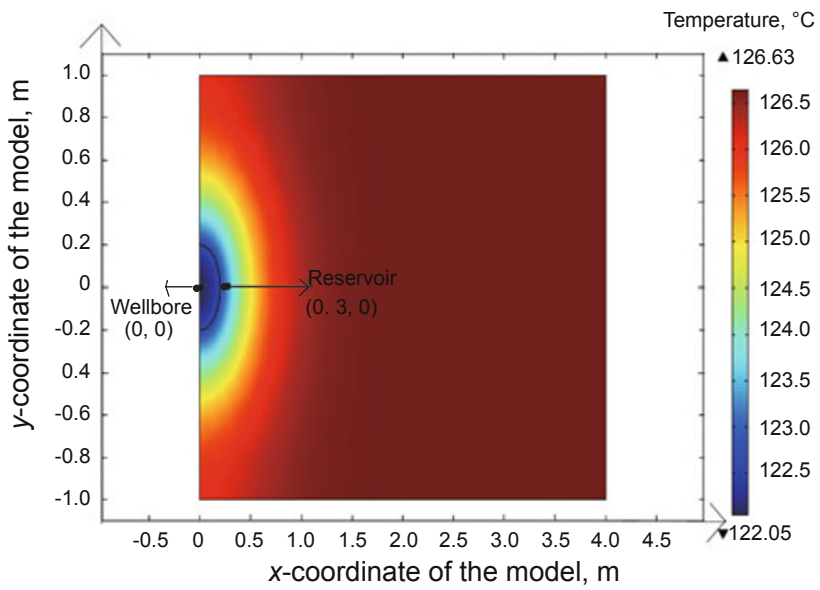

(a) 1 day

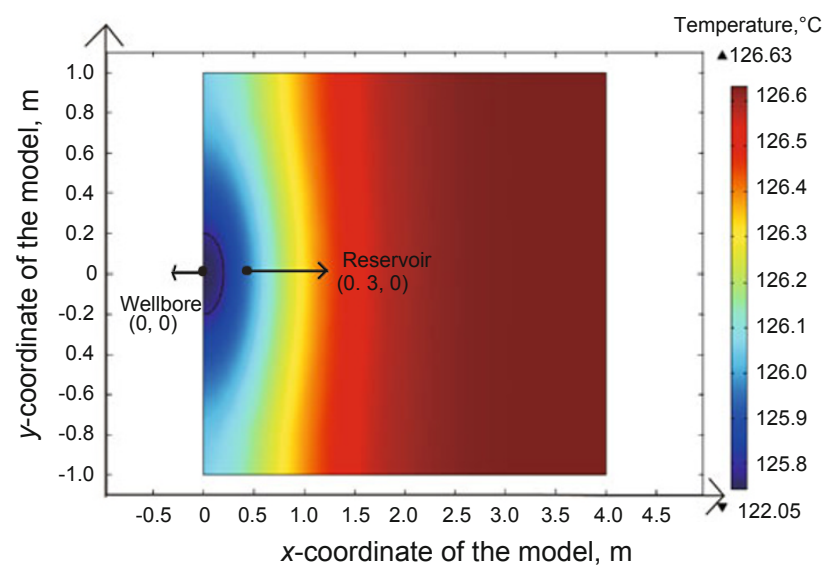

(b) 3 days

Fig. 1 Temperature distribution profiles during shut-in in the presence of lost circulation $(\varphi=0.05)$

\subsection{Numerical simulation of drilling fluid loss}

The fractured-cavernous reservoir in the Tahe oilfield exhibits strong heterogeneity, hence some of the drilling fluid often penetrates into the formation when the drill bit encounters caverns or large fractures (Wang et al, 2010; Huang et al, 2011; Kang et al, 2012). The temperature of the drilling fluid which has invaded the formation is usually low, which decreases the reservoir temperature around the 


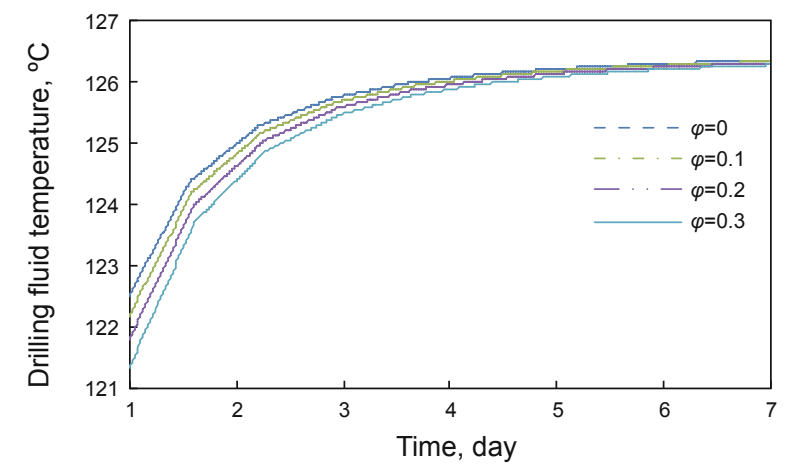

(a)

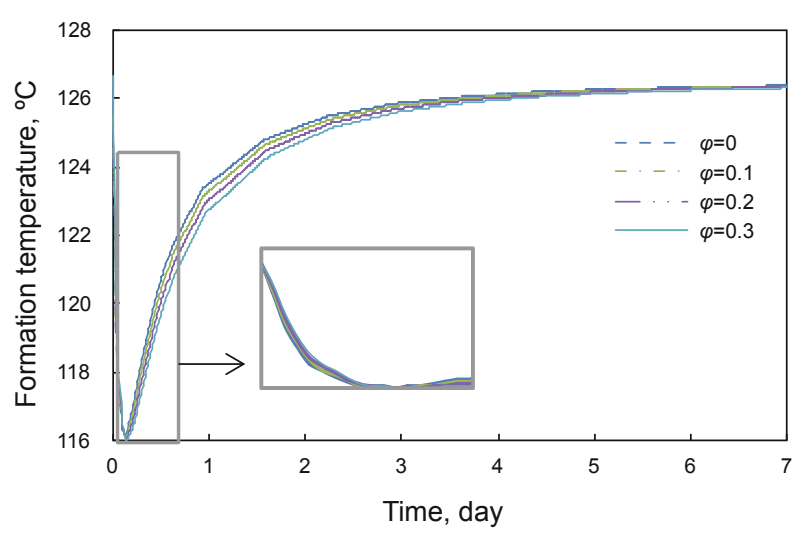

(b)

Fig. 2 Temperatures of drilling fluid and formation changing with time at different porosity values

wellbore, and the drilling fluid will be heated continuously by the formation, showing as an anomaly on the temperature curve. When the drilling fluid temperature stabilizes at close to the formation temperature, the temperature curve will have gradually returned to the normal geothermal gradient ( $\mathrm{Ma}$, 2005; Stjern et al, 2003).

We took well $\mathrm{Y}$ in the Tahe oilfield as an example to calculate the temperature change at different leakages. The data showed that the leakage interval was 5,733.66-5,738.19 $\mathrm{m}$, and the amount of leakage was $14.67 \mathrm{~m}^{3}$, the density of the drilling fluid was $1.12 \mathrm{~g} \cdot \mathrm{cm}^{-3}$, and the temperature of the leakage interval was $126.6^{\circ} \mathrm{C}$, which was measured after

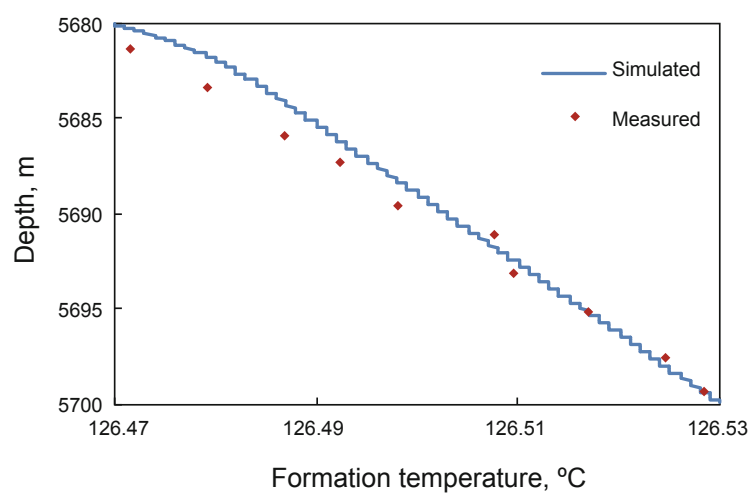

Fig. 3 Change of temperature with depth after drilling fluid invasion in well $\mathrm{Y}$ at a depth of 5680-5700 m the well was shut in for 30 days. According to the static temperature curve of the formation, the temperature of this formation interval was $126.63{ }^{\circ} \mathrm{C}$. According to the statistics of the formation pressure, the static formation pressure was 65.0 $\mathrm{MPa}$. Therefore, the reservoir formation is assumed to be a horizontal layer with dimensions of $40 \mathrm{~m} \times 60 \mathrm{~m}$ (radius $\times$ thickness), where a wellbore is located at the center and penetrates a cavern of $14.67 \mathrm{~m}^{3}$ at coordinates of $(0,0$, 24.5), as shown in Fig. 4.

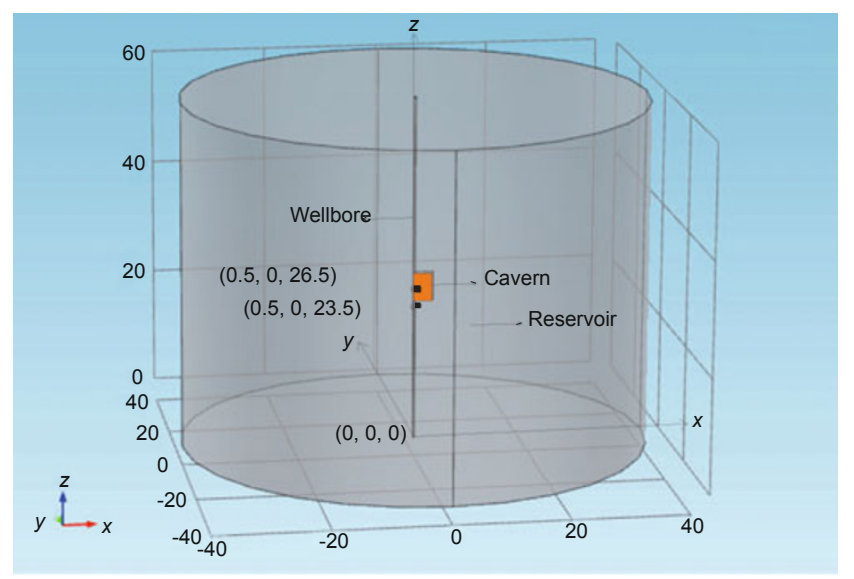

Fig. 4 Geometric model of leakage in well Y at a depth of $5733.66-5738.19 \mathrm{~m}$

When drilling to the formation interval where a cavern is encountered the drilling fluid may flow into the cavern and we use the energy conservation equation to describe the heat exchange between the cold drilling fluid and the formation. Beyond the cavern the process of drilling fluid invasion still occurs due to the formation permeability. Fig. 5 shows the log results of well Y after 30 days of shut-in. In Fig. 5, TEM(M) is the measured data, TEM is the simulated values, and we can calculate the oil production and water production by using the water holdup and flow rate, respectively. We observed the enlargement of holes at the leakage intervals, along with a sudden pressure reduction and a temperature decrease. We can see that the simulation results are well matched with the measured data, which has a significant meaning to identification of the leakage formation and forecast of the formation temperature. We select two points respectively in the cavern (at coordinates of $(0.5,0,26.5))$ and the formation (at coordinates of $(0.5,0,23.5))$ to simulate the changes of temperature with time, as shown in Fig. 6. The average simulated temperature of the cavern, which is obtained on the 30 th day, is $125.4{ }^{\circ} \mathrm{C}$, and the relative error less than $0.95 \%$. In the lost circulation process, the drilling fluid flows into the cavern, then heat transfer occurs, the formation temperature decreases rapidly and then increases slowly back to its initial value, while the drilling fluid absorbs heat from the surrounding formation, and finally a thermal balance is achieved when the drilling fluid temperature gradually rises. The reservoir temperature reduces with time first, which is caused by lost drilling fluid, and then gradually recovers, the recovery time is about twice that of the reduction time, as shown in Fig. 6(a). 


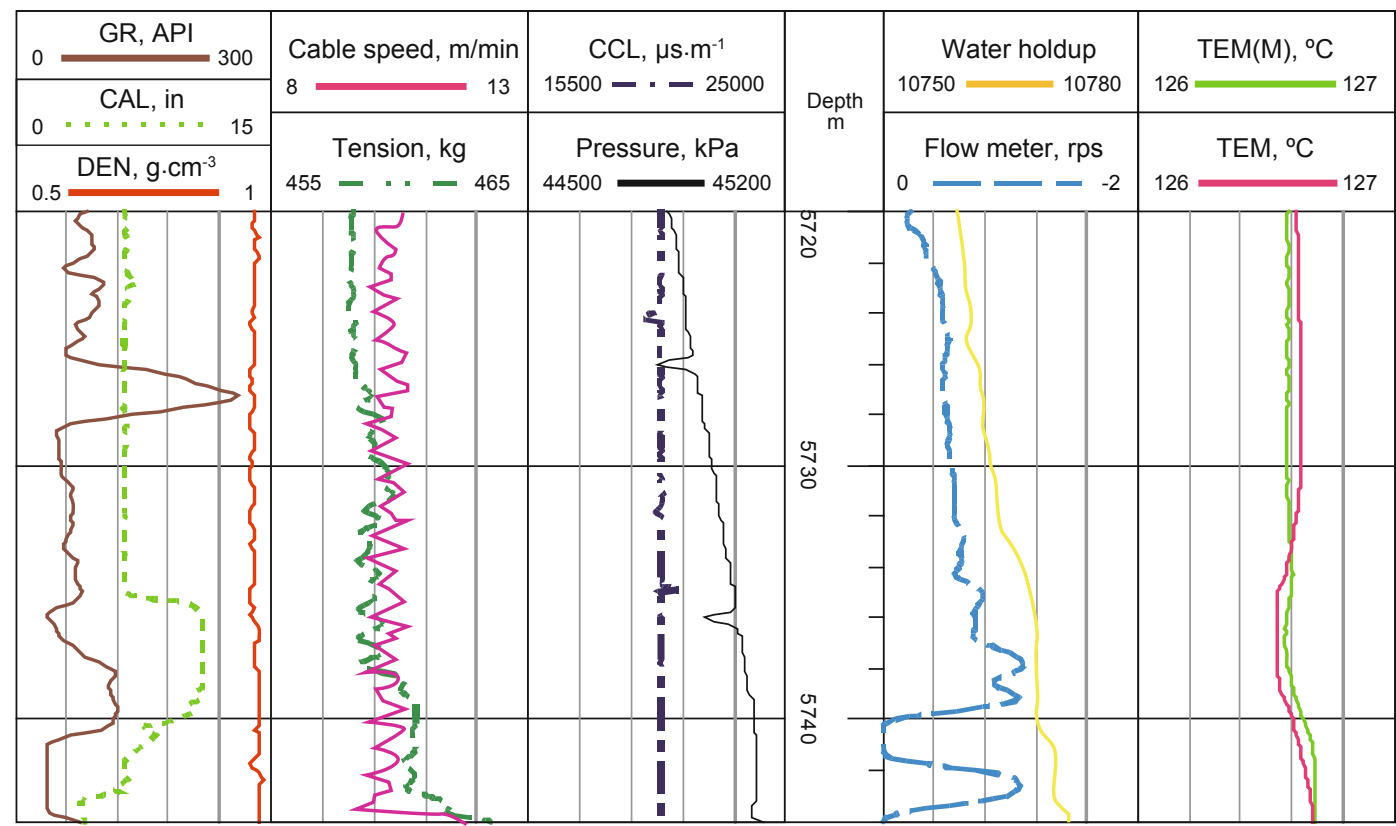

Fig. $5 \mathrm{Log}$ results of well Y with drilling fluid leakage of $14.67 \mathrm{~m}^{3}$, after 30 days of shut-in

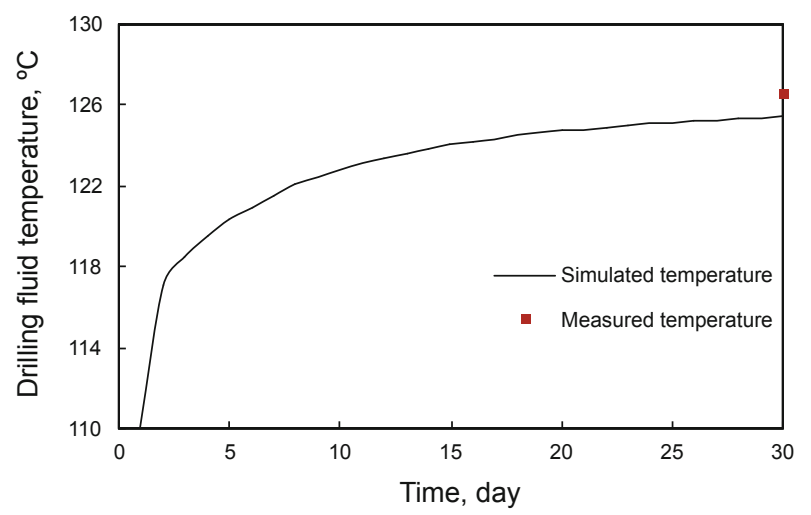

(a)

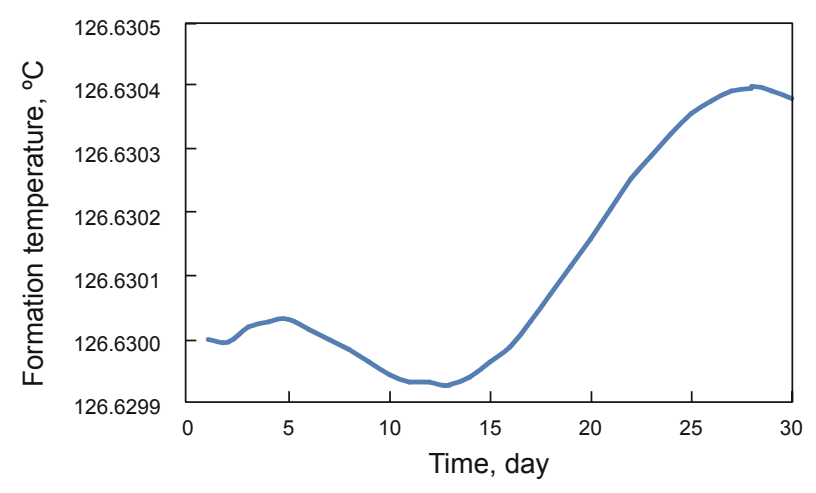

(b)

Fig. 6 Temperatures of the drilling fluid in the cavern $(0.5,0,26.5)$ and the formation $(0.5,0,23.5)$ changing with time

Fig. 7 shows the influence of porosity and cavern shape on the temperature of the drilling fluid in the cavern. Fig. 7 (a) is the temperatures of the drilling fluid in the cavern changing with time at different porosity values, where the cavern is assumed to be a cuboid with the same dimensions of $3.238 \mathrm{~m} \times 1 \mathrm{~m} \times 4.53 \mathrm{~m}(L \times W \times H)$. We see the drilling fluid temperature in the low porosity reservoir is closer to the measured value. Fig. 7(b) is the temperatures of the drilling fluid in the cavern changing with time with different cavern shapes, where the reservoir porosity is assumed to be the same. A comparison of temperature distributions in three types of cavern shape shows the drilling fluid temperature in the cuboid cavern with dimensions of $9.78 \mathrm{~m} \times 1 \mathrm{~m} \times 1.5 \mathrm{~m}$ $(L \times W \times H)$ is closer to the measured value, and the porosity has a relatively smaller influence on the simulated values than the cavern shape. Therefore, we need to choose the appropriate porosity and cavern shape in the simulation, and the simulation results are also an indication for determination of the cavern shape and location.

Fig. 8(a) is the wellbore wall temperature varying with depth when the well is shut in for 1, 5, 10, 20, $30 \mathrm{~d}$ after a total of $15 \mathrm{~m}^{3}$ of the drilling fluid is lost into the formation, where $x$ coordinate is $0.2, y$ coordinate is 0 and $z$ coordinate increases from 0 to 50 (well depth is from 5,712.69 to 5,762.69). The temperature of the drilling fluid invading the formation is usually low, so the temperature curve shows a clear anomaly. The drilling fluid which has penetrated into the formation sharply reduces the formation temperature initially. The formation then slowly recovers towards its normal temperature. Fig. 8(b) shows the relationship between the leakage amount and temperature on the 30th day. After $30 \mathrm{~d}$, the $5 \mathrm{~m}^{3}$ drilling fluid escaped into the cavern is getting close to thermal equilibrium with the formation, but the leaks of 30 $\mathrm{m}^{3}$ and $45 \mathrm{~m}^{3}$ of drilling fluid are clearly still in the process of recovery. Therefore, there is a direct relationship between well temperature, leakage and logging time. The temperature of the leaking fluid tends to increase with time, and the smaller the leakage is, the faster the heat transfers between 


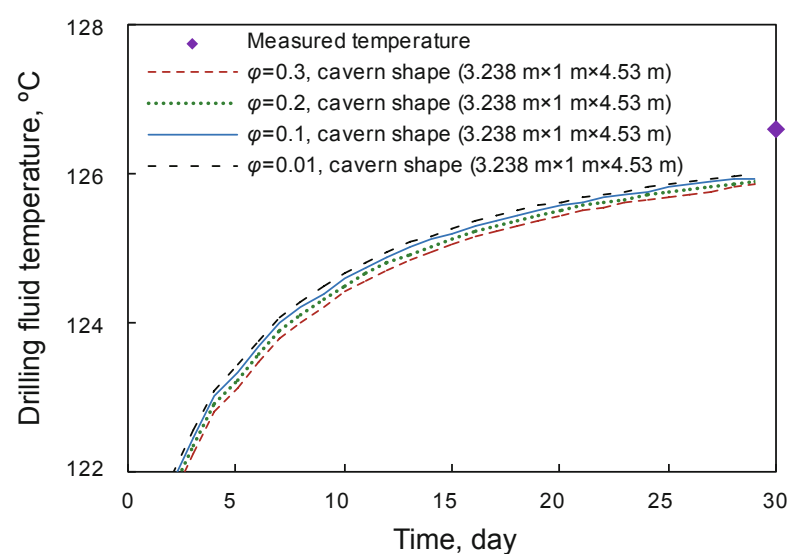

(a)

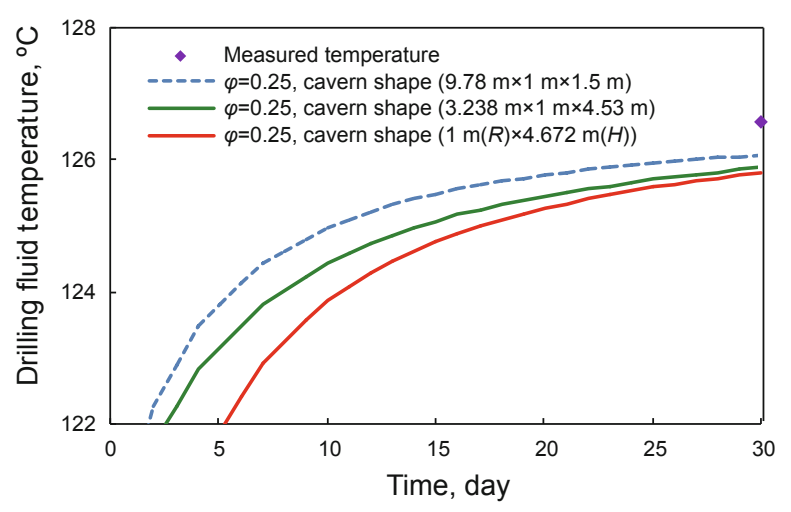

(b)

Fig. 7 Influence of porosity and cavern shape on simulated temperature of the drilling fluid in the cavern

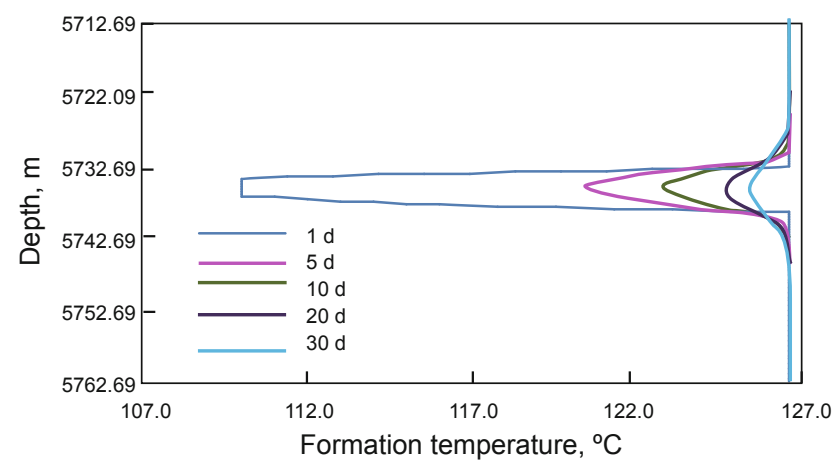

(a)

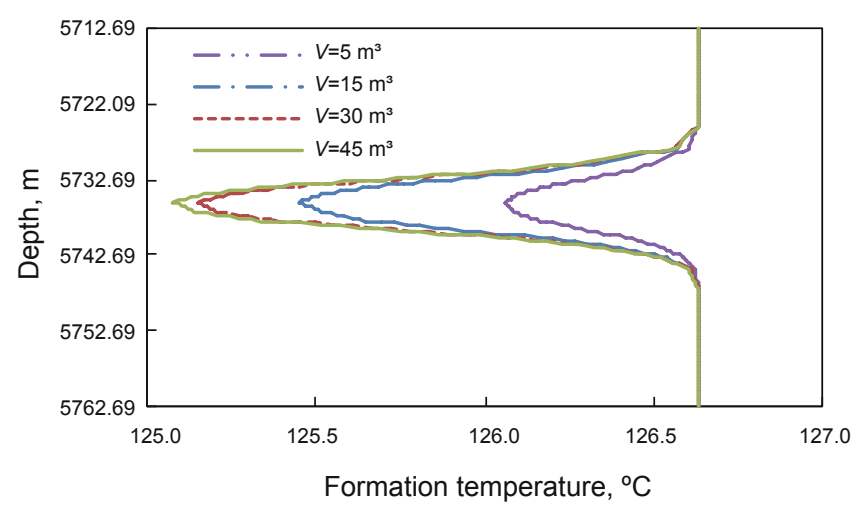

(b)

Fig. 8 Influence of logging time and leakage volume on reservoir temperature the leaked drilling fluid mud and the formation, and the faster the temperature of the lost drilling fluid increases.

In this paper, we assume that the formation is porous resulting in drilling fluid invasion and drilling fluid loss occurs when a cavern is encountered. Due to the complex distribution of fractures and the difficulty in determining the widths, fractures are not considered in this paper. However, fractures commonly exist in carbonate formations, thus there is a need for further study.

\section{Conclusions}

1) The fluid flow in the wellbore is affected by stress, where we may describe this conduit flow by the NavierStokers (NS) equation, and use Darcy's equation to describe the migration of fluid in the porous medium. The velocity fields of fluid flow in the porous reservoir and free flow in the wellbore are solved by combining both equations, and the numerical solution to the flow velocity is obtained under the given boundary conditions.

2) Substituting the obtained velocity field into the energy conservation equation gives the temperature fields of the formation and the wellbore. In this paper, heat transfer and heat convection between the wellbore and the formation are considered, giving a real multi-field coupled model. In addition, the initial and boundary conditions used to solve the equation are analyzed.

3) By simulating the formation and wellbore temperatures after invasion of the drilling fluid from the wellbore to the formation, it can be seen that when the low-temperature drilling fluid flows into the reservoir, the reservoir temperature decreases, and the drilling fluid will be heated continuously by the formation, which shows clearly on the temperature curve. When the drilling fluid temperature stabilizes close to the formation temperature, the temperature curve will gradually return to the normal geothermal gradient. Due to the drilling fluid lost into the formation, the reservoir temperature reduces with time first and then gradually recovers. The recovery time is about twice that of the reduction time, and the duration depends on multiple factors, such as the rock porosity, permeability and leakage.

4) The changes in the formation and wellbore temperatures with time are simulated after some drilling fluids have leaked into the formation. The simulated temperatures agree quite well with the measured values, which lays a foundation for potential applications of inversions of temperature curves.

\section{References}

Atkinson P, Celati R, Corsi R, et al. Thermodynamic behavior of the Bagnore geothermal field. Geothermics. 1978. 7(2-4): 185-208

Baber K, Mosthaf K and Flemisch B. Numerical scheme for coupling two-phase compositional porous-media flow and one-phase compositional free flow. IMA Journal of Applied Mathematics. 2012. 77(6): 887-909

Beirute R M. A circulating and shut-in well temperatures profile simulator. Journal of Petroleum Technology. 1991. 43(9): 1140-1146

Chin C C. Quantitative Methods in Reservoir Engineering. Gulf Professional Publishing. 2002. 235-244

Dou Q F, Sun Y F and Sullivan C. Rock-physics-based carbonate pore type characterization and reservoir permeability heterogeneity 
evaluation, upper San Andres reservoir, Permian Basin, west Texas Journal of Applied Geophysics. 2011. 74(1): 8-18

Erik H S, Frieder E, Youngseuk K, et al. Digital rock physics: effect of fluid viscosity on effective elastic properties. Journal of Applied Geophysics. 2011. 74(4): 236-241

Fang D Y and Zi R Z. On the well-posedness of inhomogeneous hyperdissipative Navier-Stokes equations. Discrete and Continuous Dynamical Systems. 2013. 33(5): 3517-3541 (in Chinese)

Feng E M, Yan G F, Hu Z R, et al. Numerical simulation and optimization for the flooding temperature fields. Acta Petrolei Sinica. 1996. 17(1): 96-102 (in Chinese)

Gao Z W, Lee K Y and Zhou Y H. Coupled thermo-mechanical analysis of functionally gradient weak/micro-discontinuous interface with graded finite element method. Acta Mechanica Solida Sinica. 2012. 25(4): 331-341

Georgiadis H G, Rigatos A P and Brock L M. Thermoelastodynamic disturbances in a half-space under the action of a buried thermal/ mechanical line source. International Journal of Solid and Structures. 1999. 36(24): 3639-3660

Huang J S, Griffiths D V and Wong S W. Characterizing natural-fracture permeability from mud-loss data. SPE Journal. 2011. 16(1): 111-114

Ji Y J, Liu J J, Chen L S, et al. Numerical computation of pressure on the thermal recovery well based on thermal-hydro-mechanical coupling. Engineering Mechanics. 2011. 28(3): 224-229 (in Chinese)

Kang Y L, You L J, Xu X, et al. Prevention of formation damage induced by mud lost in deep fractured tight gas reservoir in western Sichuan Basin. Journal of Canadian Petroleum Technology. 2012. 51(1): 4651

Lacroix S, Vassilevski Y, Wheeler J, et al. Iterative solution methods for modeling multiphase flow in porous media fully implicitly. SIMA Journal of Scientific Computing. 2003. 25(3): 905-926

Liang Z L. Convergence rate of solutions to the contact discontinuity for the compressible Navier -Stokes equations. Communications on Pure and Applied Analysis. 2013. 12(5): 1907-1926

Liu B Z and Liang B. Analysis of reservoir seepage and perforation pipeline coupling under stress based on N-S equation. Science Technology and Engineering. 2009. 9(19): 5765-5768

Liu Y Z and Wang L. Simulation and analysis of tunable performance of supercritical fluid flow in porous medium-effect of temperature gradients. Journal of Chemical Engineering of Chinese University. 2008. 6(22): 915-920 (in Chinese)

$\mathrm{Lu} \mathrm{B}$ and Wheeler M F. Iterative coupling reservoir simulation on high performance computers. Petroleum Science. 2009. 6(1): 43-50
Ma W R. The Study of Deep Acid Fracturing Technology of Fracturedvuggy Carbonate Reservoirs in the Tahe Oilfield. Ph.D. Thesis. Chengdu: Southwest Petroleum University. 2005. 35-37 (in Chinese)

Ning F L, Zhang K N, Wu N Y, et al. Invasion of water-based drilling mud into oceanic gas-hydrate-bearing sediment: one-dimensional numerical simulations. Chinese Journal of Geophysics. 2013. 56(1): 204-218 (in Chinese)

Poulsen S E, Nielsen S B and Balling N. Estimation of the equilibrium formation temperature in the presence of bore fluid invasion. Geophysical Journal International. 2012. 190(3): 1151-1561

Ramey H J. Well bore heat transmission. Journal of Petroleum Technology. 1962. 14(4): 427-435

Saenger E H, Enzmann F, Keehm Y et al. Digital rock physics: effect of fluid viscosity on effective elastic properties. Journal of Applied Geophysics. 2011. 74(4): 236-241

Sheng J C, Liu J S, Xu X C, et al. A coupled porochemothermoelastic model for borehole in shales. Engineering Mechanics. 2009. 26(12): 240-245 (in Chinese)

Stjern G, Agle A and Horsrud P. Local rock mechanical knowledge improves drilling performance in fractured formations at the Heidrun field. Journal of Petroleum Science and Engineering. 2003. 38(3): 8396

Sun S Y, Firoozabadi A and Kou J S. Numerical modeling of two-phase binary fluid mixing using mixed finite elements. Computational Geosciences. 2012. 16(4): 1101-1124

Wang S C, Jiang Y M, Zheng C J, et al. Real-time downhole monitoring and logging reduced mud loss drastically for high-pressure gas wells in Tarim Basin, China. SPE Drilling \& Completion. 2010. 25(2): 187-192

Xu S Z. The Finite Element Method in Geophysics. Beijing: Science Press. 1994. 287-296 (in Chinese)

$\mathrm{Xu} \mathrm{Y} \mathrm{B} \mathrm{and} \mathrm{Cui} \mathrm{X} \mathrm{B.} \mathrm{Analysis} \mathrm{and} \mathrm{calculation} \mathrm{method} \mathrm{of} \mathrm{temperature}$ field of steam injection well. Oil Drilling \& Production Technology. 1996. 18(4): 53-61 (in Chinese)

Zeiser T and Durst F. Efficient flow simulation on high performance computers. Computational Science and High Performance Computing. 2005. 88: 285-305

Zhang Y F, Tong M B, Dong B H, et al. Well fracturing temperature field calculation software design and realization. Journal of Yan'an University (Natural Science Edition). 2011. 30(2): 56-59 (in Chinese)

(Edited by Sun Yanhua) 